\title{
DESIGNING VIRTUAL CONSTRUCTION WORKSITE LAYOUT IN REAL ENVIRONMENT VIA AUGMENTED REALITY
}

\author{
Xiangyu Wang \\ Lecturer, Key Centre of Design Computing and Cognition \\ Faculty of Architecture \\ University of Sydney \\ Sydney NSW 2006, Australia. \\ x.wang@arch.usyd.edu.au
}

\author{
Henyi Jen \\ Assistant Professor \\ Department of Industrial Engineering \& Management \\ Yuan Ze University \\ 135 Yuan-Tung Rd., \\ Chung-Li, Taoyuan, Taiwan 320 \\ henyi@saturn.yzu.edu.tw
}

\begin{abstract}
Current construction worksite layout planning heavily relies on 2D paper media where the worksite planners sketch the future layout adjacent to their real environment. This traditional approach turns out to be ineffective and prone to error because only experienced and well-trained planners are able to generate the effective layout design with paper sketch. Augmented Reality (AR), as a new user interface technology, introduces a completely new perspective for construction worksite planning. This paper describes the concept and prototype of an AR-based construction planning tool, AR Planner with virtual elements sets and tangible interface. The focus of the paper is to identify and integrate worksite planning rules into the AR planner with the purpose of intelligently preventing potential planning errors and process inefficiency, thus maximizing the overall productivity. Future work includes refining and verifying AR Planner in realistic projects.
\end{abstract}

Keywords: Augmented Reality, construction worksite planning, construction rules, simulation, tangible user interface.

\section{INTRODUCTION}

Worksite planning of construction resources includes the design of construction worksite as well as the optimization of resource logistics. Nowadays, construction projects are in need of short duration, which necessitate a fast and flexible strategy for planning construction worksites. Current construction worksite layout planning heavily relies on 2D paper media where the worksite planners sketch the future worksite layout adjacent to their real environment. This traditional approach turns out to be ineffective and prone to error because only experienced and well-trained planners are able to generate effective construction layout design with paper scratch. This also necessitates a long training and learning period to reach an effective level of expertise.

Generally many parties (construction managers, worksite layout planner, foreman, superintendents, construction workers, etc.) from different departments/trades of the construction company are involved in the planning process. Collaboration among parties from different levels involved in the planning process cannot be well supported due to the lack of effective visual rendering inherent in the traditional paper media.

When looking at the paper media, the user could not acquire spatial impression - only a two dimensional sketch. The spatial 3D view of the entire planned scene has to be mentally constructed in human's brain. Also, the method cannot accurately reflect planning logic (e.g. representation of the active areas and safety margins) which would otherwise increases the planning certainty to a higher level. No sense of immersion could be provided in 2-D sketch. So the spatial sense of the users is not well supported and thus the frequency of mistakes during the planning process increases.

A more intuitive cooperation and visualization tool/platform is therefore necessary to accommodate the needs from all these parties involved in the planning process to support effective collaboration and communication. Augmented Reality (AR) technology suggests a new solution to the noted issues. AR technology could create an environment where the additional information generated by a computer is inserted into the user's view of a real world scene. AR technology basically augments human's perception of real world entity by inserting relevant digital information into real environment. AR allows a user to work in a real world environment while visually receiving additional computer-generated or modeled information to support the task at hand. Augmented reality environments have been applied primarily in scientific visualization and gaming entertainment in the past. In recent years, it has been explored for goal-oriented human activities like surgery, training and collaborative work. AR has been investigated in its applications in various areas of manufacturing industry such as assembly [10], quality assurance [4], industrial maintenance [8], etc. Regarding its applications in planning, AR was also explored for interior furniture layout planning [2] and manufacturing planning [5], both of which were developed based on MagicBook from HIT Lab in University of Washington [2].

The major advantage of the AR tool proposed in this paper could give construction worksite planners an easy and quick setup where even unskilled novice could easily 
capture the entire intelligent system. With the help of innovative, light-weight and inexpensive interaction and display devices, the AR interface enables the users to immerse themselves in a new reality which is augmented with computer-generated information. The introduction of $\mathrm{AR}$ as a new user interface enables a completely new approach to the development of construction worksite layout.

The proposed AR platform, called AR Planner, contributes to shortening and improving the quality of the construction worksite planning. AR Planner enables the construction worksite planner to place construction materials and machines/equipment, handling devices and the corresponding routing lines in the planned worksite. Therefore 3D-models are used to represent these objects in the virtual world. Users could move and position these items interactively to design and determine the configuration of the construction worksite.

\section{PLANNING RULES}

The integration of planning rules allows a computer-supported planning process with a high degree of faultlessness. Worksite planning rules are identified and integrated into AR Planner to intelligently assist planners in preventing possible planning errors and process inefficiency and thus maximize the overall productivity. The following major categories of planning rules are established to be integrated into AR Planner:

1) Spatial Constraints: static or dynamic site layout planning [3][7]: The site layout planning algorithms automate the allocation of macro-level spaces, which are the coarse spaces located at the construction worksite, based on user-defined qualitative adjacency constraints such as close or far between defined spaces. The major disadvantage of the mechanisms implemented in site layout planning is that it cannot generate micro-level work spaces. Examples of considerations in spatial constraints are listed below:

- Active areas and safety margins: for example, the capability range of one crane could be visualized based on the pre-determined value from the equipment specification.

- Volume of workspaces: the minimal amount of workspace which the worker needs has to be considered in advance.

- Composition and setup rules for machines: for example, certain on-site machines such as rebar bender need to be setup at a location where the path of materials flow would be optimized. The minimum and maximum distances among machines and construction equipment should also be adhered to.

2) Path planning [9]: path-planning approach involves materials flow rules, level of access to materials, work sequence, etc. This approach would be integrated into
AR Planner for material flow paths planning. The path-planning approach usually focus on providing a problem-solving strategy in a reasonable amount of time by generating a collision-free path to move a specified object from one position and orientation in the 3D computer model to another position and orientation using available manipulation mechanisms. For example, in a detailed representation of construction site, path planning will generate the specific material flow paths required to perform critical operations.

3) Environmental constraints: environmental constraints such as temperature, humidity, wetland, etc. control the planning of construction worksite to a significant extent. An interdisciplinary team composed of engineers, ecologists, aquatic and wildlife biologists, and architectural and landscape consultants need to evaluate every aspect of the construction regard to its environmental impact.

4) Resource allocation optimization strategy: indicators such as throughput, manpower requirements, inventory levels, routing behavior effects could be determined dynamically in a real time manner in an $A R$ environment. The interactive, true $3 \mathrm{D}$ animation and statistical results could be used to allocate resources appropriately, which allows justifying costs associated with work-in-process inventory, labor scheduling, equipment failure, and capacity planning.

Different graphical representations are used in the system. The representation of active areas, safety margins and workspaces is realized using bounding boxes. AR Planner uses grids to divide the virtual construction ground/site for different level of details: coarse grids for rough planning and fine grids for detailed planning. The entire model containing the construction worksite generated from AR Planner can be exported to VRML. Then the whole scene can be imported in 3D-realtime rendering systems (e.g., CAVE, workbench, head-mounted-display, etc.) for a higher degree of immersion.

\section{PROTOTYPE SYSTEM ARCHITECTURE}

The basic task of the system is the layout design of construction worksite. With a tangible user interface the user has the possibility to place and move the objects in a virtual model of a worksite. Collaboration functionality allows collaborative work among various parties. In a collaborative planning session, every user wears a head mounted display and gets a tangible interface. For each head mounted display, a PC is used to render the scene from the corresponding viewpoint. Due to the usage of different interfaces the activities of each user during the planning process could be stored. 
AR planner allows animations aside from static objects to show the behavior of the dynamic components. For example it is possible to show the movement of a truck in order to visualize its active space or to detect possible crashes with its environment. If a crane cannot reach to a desired area within its range capability, such shortfall could be automatically identified, visualized, and highlighted.

Users could plan a virtual construction worksite straightforward with planning rules built in the system to ensure the correctness of the planning alternatives. These may then be imported into an AR scene and displayed as seen in Figure 1 by AR Planner.

The exact touching of the virtual element with the paddle is difficult to achieve. Accurate placements, movements and alignments of the virtual elements in the AR scene are therefore very difficult to realize. If the planned worksite consists of a high number of 3D models which are densely placed, it is not easy to select and move a single virtual element without interfering other adjacent virtual elements.

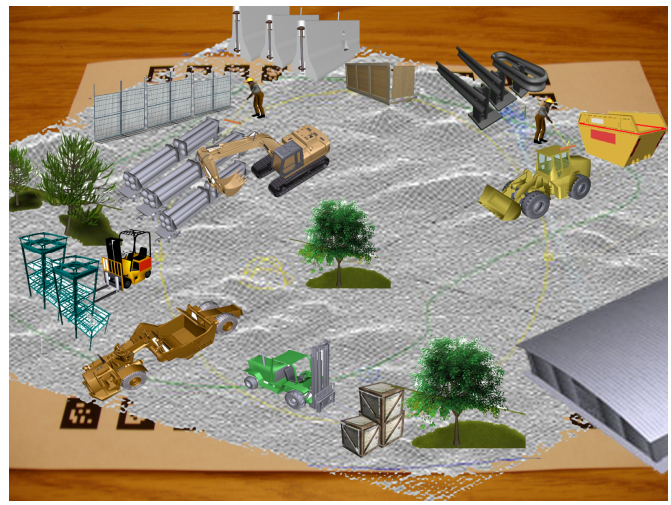

Figure 1. Virtual construction site planning on top of a real ARTag tracking marker
The system architecture of AR Planner is depicted in Figure 2 and discussed in the following sections.

\subsection{Hardware and Software}

The computing unit used is a Pentium 4 PCs with a 1.6 $\mathrm{GHz}$ processor with $512 \mathrm{MB}$ RAM and a GeForce II Ultra graphics card. Display device used is a head mounted display with a color video camera (real environment sensor) attached. The input mechanism uses the software Magic Paddle from HIT Lab, which is based on the ARToolKit software [1].

\subsection{Tracking system}

The tracking approach adopted by AR Planner is based on the ARTag system [6], a 2D fiducial marker and computer vision system for Augmented Reality. The system offers the development of table-top AR environments using markers for tracking. The fiducial markers used in the ARTag system are bi-tonal planar patterns that consist of a square border and an interior region filled with a $6 \times 6$ grid (a digital 36 bit word) of black or white cells. Figure 3 shows examples of ARTag markers. Quadrilateral contours are located in the image which may belong to the outside border of a marker. They are found in ARTag with an edge-based method, and edge pixels are threshold and linked into segments, which are in turn grouped into "squads." The four corners of the squad boundary are used to create a homography mapping to sample the marker interior. Then the 36-bit word can be extracted from a camera image of the marker once the boundary is determined. The digital word contains a unique ID number. A major advantage to ARTag's edge-based approach over similar fiducial approaches is the ability to still detect marker outlines in the presence of an occlusion.

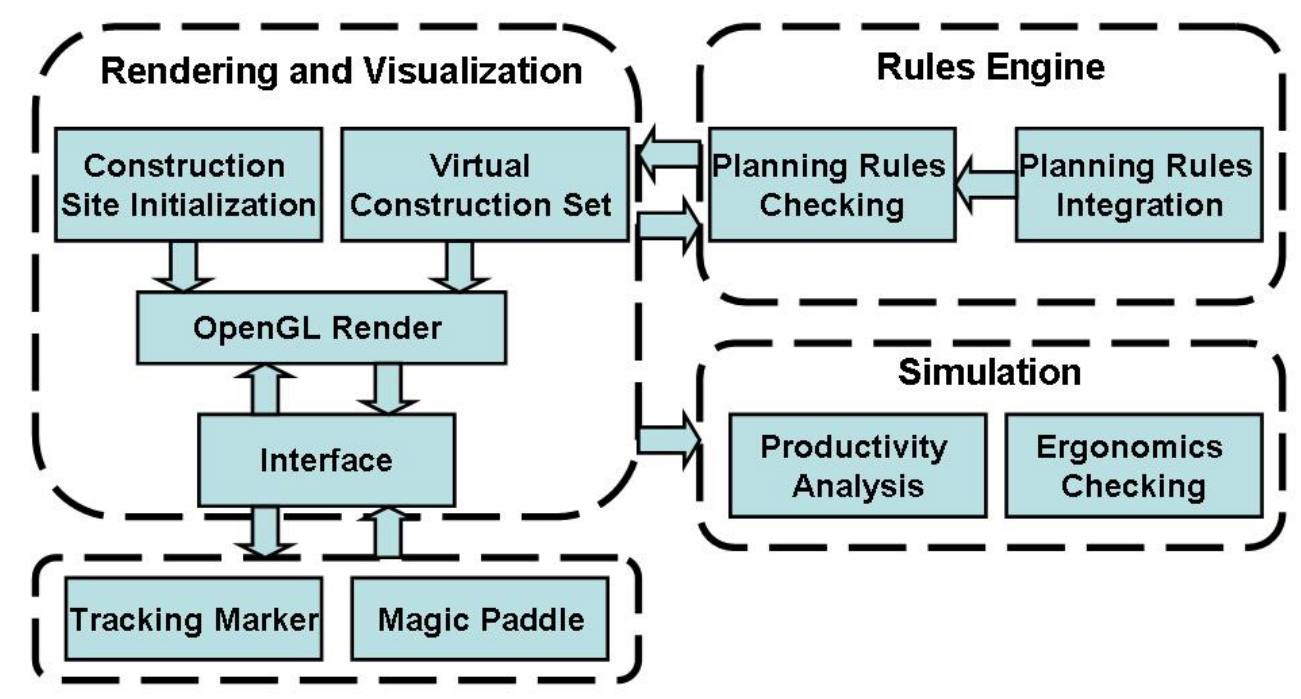

Figure 2. System Setup of AR Planner 


\subsection{Virtual Element Sets}

The virtual element sets actually provide the virtual elements that users could manipulate and interact with in the virtual construction site and they are also the elements that need to be planned. The components of the virtual element sets are 3D models of construction materials, machines, devices, equipment, and transport systems, which are created by conventional 3D CAD modeling tools. The user (equipped with a head mounted display) finds himself/herself in a scene such as in Figure 1. The components such as machines and devices of the virtual element sets are stored in a sort of book (see Figure 4). Users can select, place, and manipulate each virtual element of the virtual element sets in an easy and intuitive way using a tangible paddle. Using a paddle, modules from the virtual element sets can be brought into the existing virtual construction worksite. To be used in construction worksite planning, virtual element sets must be flexibly designed in order to enable easy adaptation to different construction worksite scenarios.



Figure 3. ARTag tracking marker

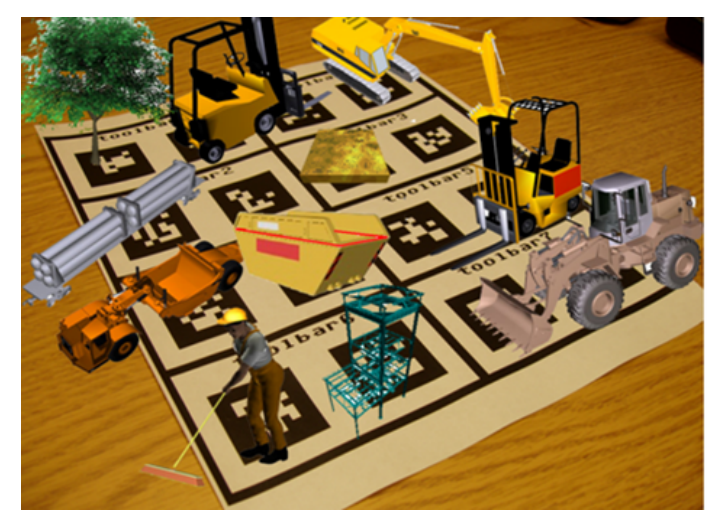

Figure 4. Virtual element set: virtual 3D models of resources overlaid on real tracking marker

\subsection{Database}

A database is to store and manage the 3D models in virtual element sets, the datasheet for the relevant description of each element and the planning rules. During the planning process the database also manages the updates of the scene. Each time a new virtual element is modeled, it is stored in the database with a datasheet. This datasheet contains information about the properties of the virtual element such as active areas, safety margins, etc. Furthermore, attributes for the planning rules could also be set in the database such as capacity ranges, connection points, etc.

\section{SIMULATION}

There are two types of simulation that could be implemented on top of the rendered planned virtual construction worksite. Apart from static layout design, the design and the optimization of materials (resources) flow path/access and logistics is extremely important. In this case the process simulation is used for designing material flows and logistics. The analytical results could be displayed in customizable numerical tables, bar graphs, pie charts, histograms, and time series graphs. Then such data could be exported into an external analysis tool such as a spreadsheet or other charting package. These analytical tools could assist in identifying and quantifying the impact of bottlenecks on planned worksite layout. Certainly ergonomic layout of the manual workplaces is also crucial for the construction crew. Elements such as tools and material can be positioned within an optimized range for the construction worker.

\section{CONCLUSIONS AND FUTURE WORK}

This paper introduced a new concept of an AR-based construction worksite planning system, AR Planner and presented the preliminary work associated with AR Planner. The prototype described in this paper shows that the use of AR-based element sets in the construction worksite planning is beneficial. The efficiency of the system mainly depends on the number and quality of construction elements available in the virtual element sets. The implementation of planning rules assists the user and prevents possible errors which normally occur during the planning phases.

Future work includes refining the AR prototype and verifying the AR system in a realistic project. This prototype will be improve/refined further to realize all the itemized functions described in this paper. Experimental validation will be implemented in future to demonstrate the effectiveness of AR Planner, especially the benefits for novice planners. The benchmark method compared will be paper media sketch. After the configuration is designed with the AR-based element sets, it can be verified in the real environment using an outdoor wearable AR-system. This system would blend planned layout of all relevant virtual objects/components into the real construction worksite. In general the tracking of the user is the main problem. 


\section{ACKNOWLEDGEMENTS}

The described prototype is implemented using the new version of the ARTag system by Dr. Mark Fiala in National Research Council Canada, Institute for Information Technology.

\section{REFERENCES}

[1] M. Billinghurst, K. Imamoto, H. Kato, and K. Tachibana (2001). "Magic Paddle: A Tangible Augmented Reality Interface for Object Manipulation." Proceedings of the Second International Symposium on Mixed Reality, 194-195.

[2] M. Billinghurst, H.Kato, and I. Poupyrev (2001). "The MagicBook-Moving Seamlessly between Reality and Virtuality," IEEE Computer Graphics and Applications, 21 (3), 6-8.

[3] H.Y. Choo and I. Tommelein (1999). "Space Scheduling Using Flow Analysis." IGLC-7, Berkeley, CA, 299-311.

[4] D. Curtis, D. Mizell, P. Gruenbaum, A. Janin (1999). "Several Devils in the Details: Making an AR Application Work in the Airplane Factory." Proceedings of the international workshop on Augmented Reality: placing artificial objects in real scenes, $47-60$.

[5] F. Doil, W. Schreiber, T. Alt, and C. Patron (2003). "Augmented Reality for manufacturing planning." Proceedings of International Immersive Projection Technologies Workshop, J. Deisinger, A. Kunz (Editors), 71-76.

[6] M. Fiala (2004). "ARTag Revision 1, a fiducial marker system using digital techniques," NRC/ERB-1117, NRC Publication Number: NRC 47419, November 24.

[7] T. Hegazy and E. Elbeltagi (1999). "EvoSite: Evolution-Based Model for Site Layout Planning." Journal of Computing in Civil Engineering, 13 (3), 198-206.

[8] H. Lipson, M. Shpitalni, F. Kimura and I. Goncharenko (1998). "Online Product Maintenance by Web-Based Augmented Reality." Proceedings of CIRP Design Seminar on New Tools and Workflows for Product Development.

[9] A. Morad, A. Cleveland, Y Beliveau, V. Francisco, and S. Dixit (1992). "Path-Finder: AI-based Path Planning System." Journal of Computing in Civil Engineering, 6 (2), 114-128.

[10] D. Reiners, S. Stricker, G. Klinker and S. Müller (1999). "Augmented Reality for Construction Tasks: Doorlock Assembly." Proceedings of the international workshop on Augmented Reality: placing artificial objects in real scenes, Natick, MA. 